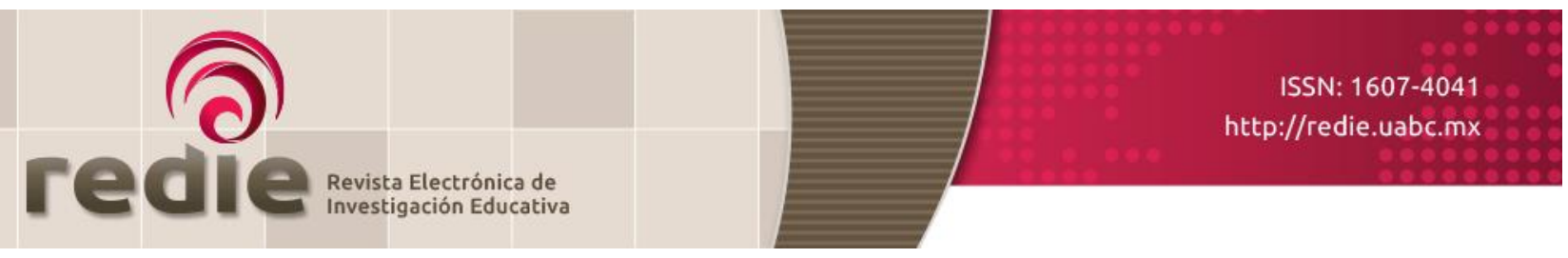

Vol. 20, Núm. 1, 2018

\title{
Incidencia del trabajo colaborativo docente en la enseñanza y el aprendizaje de la escritura académica ${ }^{1}$
}

\author{
Impact of Collaborative Work by Teachers in Teaching \\ and Learning Academic Writing
}

\author{
Karen Shirley López-Gil (*) karens@javerianacali.edu.co \\ Violeta Molina Natera (*) vmolina@javerianacali.edu.co \\ (*) Pontificia Universidad Javeriana Cali \\ (Recibido: 17 de agosto de 2016; Aceptado para su publicación: 27 de octubre de 2016) \\ Cómo citar: López-Gil, K. S. y Molina, V. (2018). Incidencia del trabajo colaborativo docente en la enseñanza y el aprendizaje de la \\ escritura académica. Revista Electrónica de Investigación Educativa, 20(1), 1-13. https://doi.org/10.24320/redie.2018.20.1.1477
}

\section{Resumen}

Esta investigación buscaba analizar la incidencia de una estrategia de trabajo colaborativo, entre profesores de lenguaje y de otras áreas, en la enseñanza y el aprendizaje de la escritura en cuatro asignaturas de los programas de Medicina, Administración, Derecho e Ingeniería de Sistemas de una universidad privada colombiana. Se siguió un diseño mixto de investigación, en el que se realizó una encuesta sobre representaciones y prácticas de escritura a 86 estudiantes, una entrevista semiestructurada a docentes y un grupo de discusión con los alumnos. Se evidenciaron cambios en las prácticas de orientación y evaluación de la escritura de los profesores, así como transformaciones en la representación de la función de la escritura y en las prácticas de composición escrita de los estudiantes. A partir de estos hallazgos, se propone el trabajo colaborativo docente como una estrategia institucional que puede potenciar la enseñanza y el aprendizaje de la escritura a través del currículo.

Palabras clave: Escritura, universidad, formación docente, aprendizaje.

\section{Abstract}

This study sought to analyze the impact of a collaborative work strategy implemented between language teachers and teachers from other areas in teaching and learning writing in four courses from the medicine, administration, law and systems engineering programs in a private university in Colombia. A mixed-method research design was used, which included a survey conducted with 86 students on writing practices and representations, a semi-structured interview with teachers, and a discussion group with students. Changes were found in teachers' guidance and writing assessment practices and in students' representations of the function of writing and written composition practices. On the basis of these

\footnotetext{
${ }^{1}$ Proyecto de investigación financiado por la Pontificia Universidad Javeriana Cali (Colombia), convocatoria Capital Semilla (2014-2015), código 00004023.
} 
findings, collaborative work by teachers is proposed as an institutional strategy that can enhance the teaching and learning of writing through the curriculum.

Keywords: Writing, University, Teacher education, Learning.

\section{Introducción}

La escritura es reconocida como una competencia fundamental en la formación de los estudiantes universitarios. En las últimas décadas se evidencia un creciente interés por las formas de enseñar y de aprender a escribir en las universidades de América Latina. Perspectivas como la de McLeod, Miraglia, Soven y Thaiss (2001) y Carlino (2005) han puesto de manifiesto que los modos de escribir en educación superior difieren considerablemente de lo que se hace en otros niveles educativos y contextos. Desde estas perspectivas, la escritura se concibe como heterogénea (Olson, 2013), en tanto que varía en las disciplinas de estudio y en los distintos momentos del proceso formativo.

Por lo anterior, la escritura requiere de un acompañamiento explícito en la universidad. Este acompañamiento no se limita a los docentes de lenguaje, pues al configurarse unas formas de escribir particulares en las disciplinas se requiere también de orientación por parte de los docentes de otras áreas. El principal desafíos que los profesores universitarios se han formado como especialistas en sus campos de conocimiento, pero suelen dominar de forma tácita los modos discursivos propios de sus disciplinas, razón por la cual el apoyo de docentes expertos en lenguaje puede potenciar la orientación de la escritura a través del currículo de manera más efectiva (Paretti, 2011).

Una manera de concretar este apoyo es a través del trabajo colaborativo docente en escritura, entendido como un conjunto de actividades articuladas entre el docente de lengua y el docente de la disciplina que tiene como objetivo común facilitar los aprendizajes de los estudiantes mediante la escritura y acercarlos a las formas de comunicación específicas de sus áreas de conocimiento. El trabajo colaborativo tiene como característica el establecimiento de una meta conjunta, que es difícil de lograr de manera individual (Jacobs, 2007; Lavié, 2009). Este tipo de estrategia se enmarca en procesos de interformación, en los que los miembros aprenden mutuamente y reconfiguran el objeto de estudio a partir del diálogo, la participación y la co-construcción del conocimiento. En las prácticas interformativas se busca que haya una transformación tanto de las representaciones como de las prácticas cotidianas de los docentes en las aulas (García y Vaillant, 2001; Imbernón, 2007).

Distintas experiencias de trabajo colaborativo entre docentes de lengua y docentes de otras áreas han evidenciado que su principal característica es la negociación (Airey, 2010; Harran, 2010; Jacobs, 2007; Kuriloff, 2000; Leibowitz et al., 2011; Marshall et al., 2011; Moyano, 2010). Hay un reconocimiento de los saberes y experiencias de cada profesor, por lo que las relaciones suelen ser horizontales y simétricas, con una clara delimitación de los roles que cada integrante asume. En el ámbito universitario esta estrategia es pertinente en asignaturas de escritura intensiva, es decir, en cursos propios de las disciplinas en los que se usa la escritura como herramienta de aprendizaje y comunicación.

A partir de esos planteamientos, esta investigación analizó la incidencia que tuvo una estrategia de trabajo colaborativo entre docentes de las disciplinas y docentes de lengua en la enseñanza y el aprendizaje de la escritura en cuatro asignaturas disciplinares en una universidad privada colombiana.

\section{Metodología}

Esta investigación tenía, por una parte, una intención formativa al proponer una estrategia de trabajo entre el profesorado que impactara en las formas de enseñar y de aprender a escribir en la universidad; y por otra parte, una intención investigativa al documentar la incidencia de la estrategia en las representaciones y prácticas de los docentes y los estudiantes (McMillan y Schumacher, 2005).

El diseño de la investigación empleó aproximaciones cuantitativas y cualitativas, así como distintas técnicas de recolección y análisis de la información (Tashakkori y Teddlie, 2003). 
En cuanto a la estrategia, los profesores de las disciplinas participaron en un seminario-taller en el que se reflexionó sobre los propósitos y formas de usar la escritura en la universidad (Gottschalk y Hjortshoj, 2004; Young, 2006); luego se estableció la estrategia de trabajo colaborativo, en la que cada profesor de las asignaturas era acompañado por un docente de escritura durante un semestre académico -la propuesta de acompañamiento se planteó a partir de distintas experiencias (Harran, 2010; Leibowitz et al., 2011; López-Gil, Gómez y Pedraza, 2012; Moyano, 2010) y del ajuste a las características propias del contexto institucional. La dupla docente de lenguaje/docente de la disciplina planeó conjuntamente las asignaciones de escritura, identificó formas de escribir propias de las disciplinas, diseñó guías y estableció criterios de evaluación para las asignaciones.

En el curso "Procesos de ingeniería de software" los profesores diseñaron dos asignaciones: la escritura de un miniproyecto en formato de artículo científico y la escritura de un proyecto de desarrollo de software. El proyecto incluía, a su vez, la escritura de un manual técnico y un manual de usuario. En la asignatura de Medicina, "Microbiología y cine", la principal asignación fue la escritura de un texto argumentativo sobre las implicaciones sociales y éticas del VIH-SIDA. De igual modo, en la asignatura "Creatividad e idea de negocio" se solicitó la escritura de un texto argumentativo que debía justificar la creación de un producto innovador. Por último, en la asignatura "Títulos valores", de Derecho, los estudiantes escribieron un texto argumentativo en el que defendían o no la validez de un pagaré. En la tabla I se evidencian las características del trabajo colaborativo entre los profesores.

Tabla I. Características del trabajo colaborativo propuesto en las asignaturas

\begin{tabular}{|c|c|c|c|c|}
\hline Características / Asignatura & $\begin{array}{l}\text { Procesos de } \\
\text { ingeniería de } \\
\text { software }\end{array}$ & $\begin{array}{l}\text { Microbiología } \\
\text { y cine }\end{array}$ & $\begin{array}{l}\text { Creatividad e } \\
\text { idea de negocio }\end{array}$ & Títulos valores \\
\hline $\begin{array}{l}\text { Momento del trabajo } \\
\text { colaborativo entre profesores }\end{array}$ & $\begin{array}{l}\text { A lo largo del } \\
\text { semestre }\end{array}$ & $\begin{array}{l}\text { Un momento } \\
\text { específico del } \\
\text { semestre }\end{array}$ & $\begin{array}{l}\text { Un momento } \\
\text { específico del } \\
\text { semestre }\end{array}$ & $\begin{array}{l}\text { A lo largo del } \\
\text { semestre }\end{array}$ \\
\hline $\begin{array}{l}\text { Número de sesiones de trabajo } \\
\text { colaborativo con profesores de } \\
\text { lenguaje }\end{array}$ & 14 & 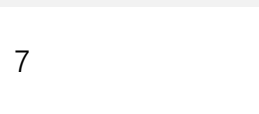 & (1) & 13 \\
\hline $\begin{array}{l}\text { Principal asignación de } \\
\text { escritura durante el curso }\end{array}$ & $\begin{array}{l}\text { Miniproyecto y } \\
\text { proyecto de } \\
\text { desarrollo de } \\
\text { software }\end{array}$ & $\begin{array}{l}\text { Texto } \\
\text { argumentativo } \\
\text { sobre las } \\
\text { implicaciones } \\
\text { sociales del VIH }\end{array}$ & $\begin{array}{l}\text { Texto } \\
\text { argumentativo } \\
\text { que justifica la } \\
\text { creación de un } \\
\text { producto }\end{array}$ & $\begin{array}{l}\text { Texto } \\
\text { argumentativo } \\
\text { sobre la validez } \\
\text { de un pagaré }\end{array}$ \\
\hline $\begin{array}{l}\text { Diseño de guías escritas para las } \\
\text { actividades }\end{array}$ & Sí & Sí & Sí & Sí \\
\hline $\begin{array}{l}\text { Diseño de rúbricas de } \\
\text { evaluación }\end{array}$ & Sí & Sí & No & Sí \\
\hline $\begin{array}{l}\text { Asesorías a estudiantes para el } \\
\text { desarrollo de los textos }\end{array}$ & Sí & Sí & Sí & Sí \\
\hline $\begin{array}{l}\text { Solicitó entregas previas de } \\
\text { avances o borradores }\end{array}$ & Sí & Sí & Sí & Sí \\
\hline $\begin{array}{l}\text { Responsable de la revisión de } \\
\text { las entregas previas }\end{array}$ & Profesor & Profesor & Profesor & $\begin{array}{l}\text { Estudiantes } \\
\text { (pares) }\end{array}$ \\
\hline $\begin{array}{l}\text { Talleres de apoyo en clase para } \\
\text { trabajar aspectos de la escritura }\end{array}$ & No & $\begin{array}{l}\text { Tesis, citas y } \\
\text { referencias, } \\
\text { redacción }\end{array}$ & $\begin{array}{l}\text { Texto } \\
\text { argumentativo }\end{array}$ & No \\
\hline
\end{tabular}

Fuente: elaboración propia.

El trabajo colaborativo varió de acuerdo con las características de los profesores participantes y de la naturaleza de los cursos. En el curso de Ingeniería, por ejemplo, la principal asignación fue la escritura de un proyecto con distintas entregas y esto implicó un trabajo continuo entre profesores, mientras que en la asignatura de Medicina la asignación se dio en un momento específico del semestre y requirió menos negociaciones por parte de los docentes. En la estrategia se buscó que las asignaciones diseñadas aportaran a la consecución de los objetivos de aprendizaje en cada uno de los cursos, y permitieran a los estudiantes apropiarse de los contenidos y aproximarse a los modos discursivos propios de sus áreas. Se 
enfatizó en la definición de situaciones comunicativas ajustadas al momento formativo de los alumnos: en la asignatura de Ingeniería el proyecto estaba dirigido a profesionales e incluía la escritura de un manual de usuario para un público no especializado; en la asignatura de Medicina el texto argumentativo estaba dirigido a profesionales de la salud; en Administración a posibles inversionistas y en Derecho a un juez.

Para apoyar a los estudiantes en la escritura de estos textos los profesores diseñaron guías que incluyeron orientaciones respecto a los propósitos de las actividades, procedimientos sugeridos, contenidos esperados y estructuras de los documentos. De igual forma, para la evaluación se definieron procesos que implicaran la entrega de avances y revisiones previas de los textos (por el profesor o entre pares) y en tres de los cuatro cursos se crearon rúbricas con criterios claros de evaluación y descripción de niveles de desempeño. Otros apoyos establecidos por los profesores fueron asesorías individuales y talleres en clase sobre temas que generaban inquietudes a los alumnos, por ejemplo, el planteamiento de la tesis o los formatos de citación.

Para realizar el estudio se estableció un muestreo no probabilístico intencional (McMillan y Schumacher, 2005). El principal criterio de selección de la muestra fue que los docentes encargados de los cursos participaran en el seminario-taller y en la estrategia de trabajo colaborativo con los profesores de lenguaje. En los cuatro cursos participaron 86 estudiantes.

Se utilizaron tres técnicas de recolección de información:

Encuesta: compuesta por 22 preguntas, centradas en identificar 1) las representaciones de los estudiantes sobre el papel de la escritura en la universidad, ${ }^{2}$ 2) sus características, 3) las propias prácticas de composición escrita y 4) las prácticas de orientación de los docentes. ${ }^{3}$

Entrevistas semiestructuradas a docentes: se establecieron preguntas sobre los cambios identificados en sus propias prácticas sobre la orientación y la evaluación de la escritura, así como las transformaciones evidenciadas en los estudiantes a partir de la estrategia de trabajo colaborativo docente.

Grupo de discusión (Ibáñez, 1986): tuvo como finalidad profundizar en los resultados obtenidos a partir de la encuesta. Las preguntas que orientaron la discusión se relacionaron con las representaciones de los estudiantes respecto al papel y las características de la escritura en la universidad, sus prácticas de composición escrita y la incidencia que en éstas tuvieron las orientaciones, así como las formas de evaluación planteadas por los docentes a partir de la estrategia de trabajo colaborativo.

\section{Resultados}

Los resultados se organizaron, a partir de la técnica de análisis de contenido (Bardin, 1996; LópezNoguero, 2002) en dos grandes categorías: incidencia de la estrategia de trabajo colaborativo en la enseñanza de la escritura e incidencia en el aprendizaje.

\subsection{Incidencia en la enseñanza}

Esta categoría da cuenta de los cambios evidenciados en las prácticas de enseñanza de la escritura, divididas en dos subcategorías: orientación y evaluación.

\footnotetext{
${ }^{2}$ La noción de representaciones sobre la escritura se retomó de Bocca y Vasconcelo (2009), quienes las entienden como los "sistemas interpretativos que pueden generar actitudes frente a la escritura (...) orientadas hacia sus prácticas y relaciones contextuales" (p. 23), y de Carlino (2006), quien las define como las ideas que fundamentan y justifican las acciones que se asumen respecto a la escritura e incluyen las formas de entender su naturaleza, función, enseñanza, aprendizaje y evaluación.

${ }^{3}$ Se entiende por prácticas todas aquellas acciones que se repiten cotidianamente, que son constantes en el tiempo y que se configuran a partir de las características de los sujetos y de los contextos en los cuales se desenvuelven (Pérez y Rincón, 2013).
} 
Prácticas de orientación de la escritura por parte de los docentes. Las prácticas de orientación se refieren a las acciones planteadas por los docentes para acompañar de forma efectiva la escritura en sus cursos. En las prácticas anteriores al trabajo colaborativo se encontró que uno de los docentes entregaba las consignas por escrito, mientras que los otros tres las presentaban de forma oral. Durante la estrategia los profesores diseñaron guías que detallaban los propósitos de las actividades, los procedimientos a seguir y las características discursivas del producto a entregar.

En la encuesta a los estudiantes se les preguntó por la efectividad de las guías escritas en los cursos, comparando las características de las orientaciones de los profesores de las cuatro asignaturas con trabajo colaborativo (TC) con otros cursos de la universidad. Los estudiantes consideraron que las guías entregadas en los cursos con tc son más efectivas que las que reciben en otras asignaturas (figura 1).

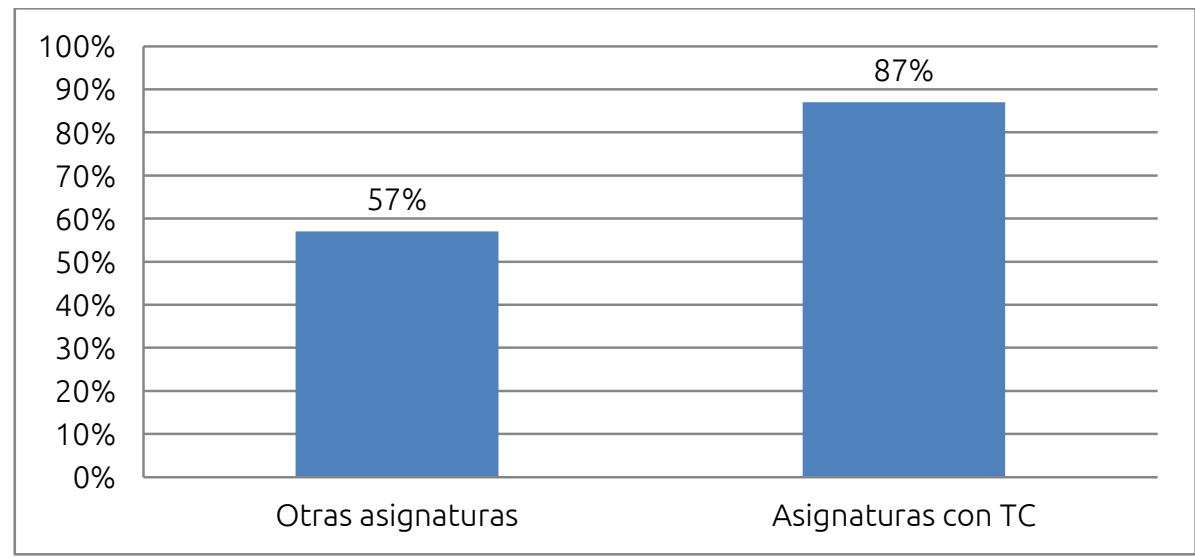

Fuente: elaboración propia.

Figura 1. Efectividad de las guías escritas.

En el grupo de discusión los estudiantes indicaron que la mayoría de docentes en otros cursos plantean consignas orales para las asignaciones de escritura. Por otra parte, destacan las ventajas de contar con guías detalladas para el desarrollo de las actividades, pues pueden tener más claro qué se espera de sus textos:

Creo que es pertinente tener la guía porque se muestra el orden o el proceso que debemos llevar a cabo y también nos dice lo que se espera que tenga el texto (...) (GDE2). ${ }^{4}$

La mayoría piden los trabajos y lo dicen en clase, pero uno no tiene claro qué es lo que esperan que hagamos. Si entregan una guía se puede saber qué espera el profesor y uno siempre la tiene a la mano, no se le olvida. (GDE3).

De igual modo, en las entrevistas a profesores se evidencia la importancia de la guía como mediadora del proceso de enseñanza y aprendizaje de la escritura.

Yo siempre he tratado de darles las indicaciones por escrito, pero mis guías no eran tan detalladas; no tenían, por ejemplo, el propósito de la actividad para que ellos le encontraran sentido. Ahora incluyo también aspectos del proceso y no sólo del producto escrito. (P1). ${ }^{5}$

Aunque no todos cumplen con lo solicitado, la guía funciona como un contrato. Cuando les comento algo los hago volver sobre la guía y se dan cuenta que todo estaba allí. (P4).

\footnotetext{
${ }^{4}$ Los estudiantes que participaron en el grupo de discusión se identifican con las letras GDE y están numerados del 1 al 7.

${ }^{5}$ Los profesores de las cuatro asignaturas se identifican con la letra P y están numerados del 1 al 4.
} 
En las prácticas de orientación se identificó también que los cuatros profesores solían realizar asesorías en las tareas de escritura, este apoyo se mantuvo durante la estrategia de trabajo colaborativo. Respecto a las asesorías, 65\% de los estudiantes consideró que fueron efectivas en las asignaturas con tc y $47 \%$ en otras asignaturas (figura 2).

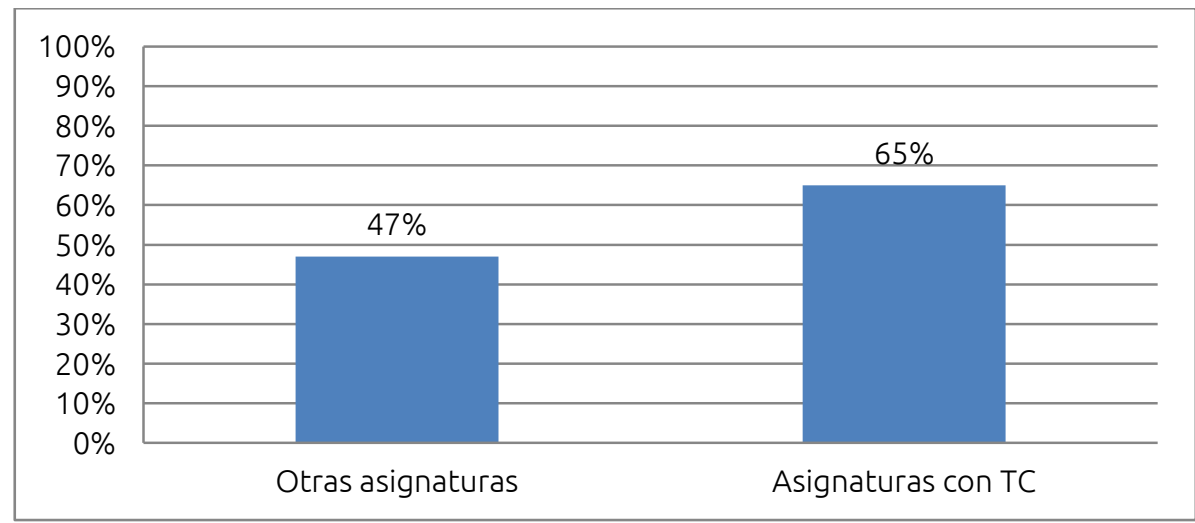

Fuente: elaboración propia.

Figura 2. Asesorías centradas en escritura.

En el grupo de discusión, los estudiantes indicaron que las asesorías son frecuentes en las distintas asignaturas en la universidad, pero que en general estas se centran en aspectos más globales del trabajo y no en la escritura.

[las asesorías] son importantes porque van indicando cómo va el trabajo y se aclaran las dudas con el docente, pero creo yo que casi siempre son como para verificar cómo vamos, las dudas, no tanto para leer y ayudar a darle forma al texto. (GDE3).

Prácticas de evaluación de la escritura por parte de los docentes. Esta categoría se refiere a cómo se evalúan las tareas de escritura en las asignaturas. En los resultados destacan dos aspectos de la evaluación que fueron producto de la estrategia de trabajo colaborativo docente: 1) la evaluación formativa, en la que se permitió la entrega de borradores o avances, y 2) la descripción de los criterios de evaluación a través de rúbricas.

En la encuesta a estudiantes se encontró que es poco frecuente en otras asignaturas que los profesores pidan avances y los devuelvan con observaciones y posibilidades de ajuste (22\%), mientras que en las asignaturas con TC el 93\% identificó que esta era una práctica común (ver figura 3). Antes de entregar el texto, los profesores pidieron a los estudiantes que hicieran una planeación y al menos un borrador que fue revisado por el propio profesor (en los casos de Ingeniería, Administración, Medicina) o por los compañeros (en el caso de Derecho). Después de la revisión y de los ajustes se hacía la entrega definitiva. 


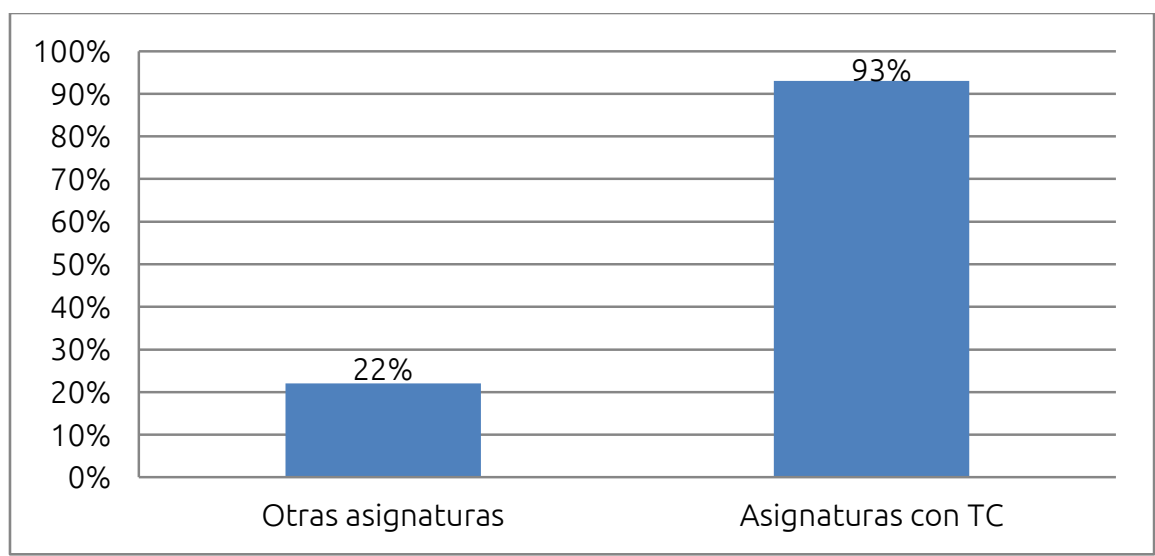

Fuente: elaboración propia.

Figura 3. Evaluación formativa: avances y ajustes de los textos.

Al respecto, en el grupo de discusión los estudiantes valoraron la presentación de avances y la realización de ajustes como una oportunidad para aprender:

(...) las observaciones son muy puntuales y cuando se hacen los cambios nos vamos dando cuenta de que tal vez no habíamos entendido bien un concepto o un procedimiento y allí es donde se va transformando lo que sabemos. (GDE1).

Me siento más tranquilo cuando se presenta un avance porque así me doy cuenta de si voy bien o tengo que encaminarme por otro lado... eso es una oportunidad de aprendizaje. (GDE5).

Рara los profesores, aunque la revisión de avances implica mayor inversión de tiempo, permite que las versiones definitivas de los textos se ajusten más a lo requerido.

Creo que la revisión no calificable del borrador fue muy importante. Me demoré mucho más, pero ellos valoraron los comentarios y siguieron las recomendaciones, lo que hizo que la revisión de la entrega final fuera más sencilla y también satisfactoria... (P2).

Finalmente, se indagó con los estudiantes acerca del uso y la pertinencia de las rúbricas de evaluación. Cabe recordar que en una de las cuatro asignaturas con TC no se compartieron criterios específicos de evaluación a través de una rúbrica, pero sí se establecieron criterios generales en la guía escrita de la asignación.

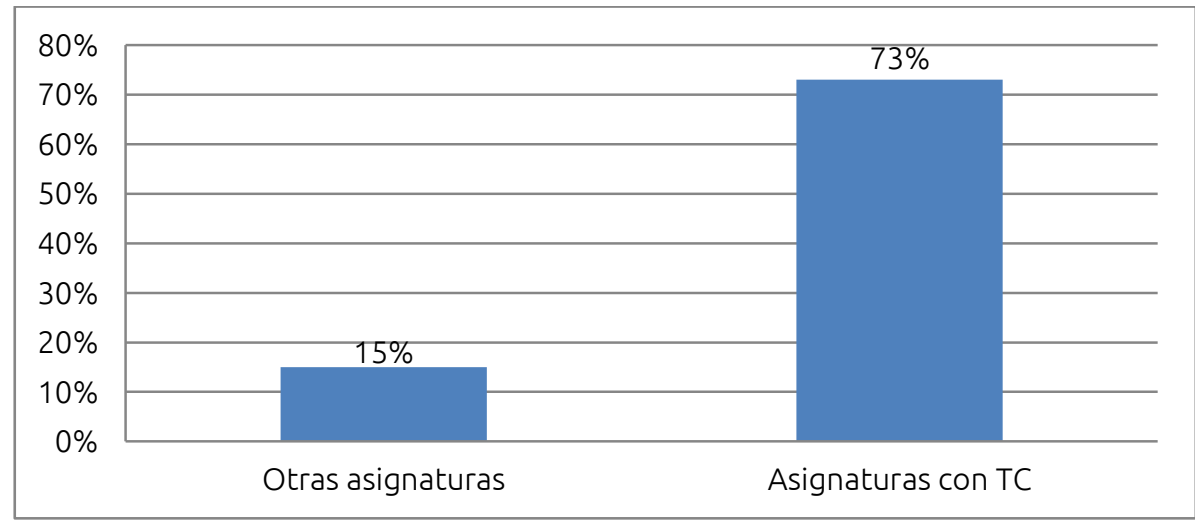

Fuente: elaboración propia.

Figura 4. Uso de rúbricas de evaluación. 
Como se evidencia en la figura 4, la práctica de evaluación a través de rúbricas no es frecuente en otras asignaturas cursadas por los estudiantes (15\%). En el grupo de discusión los alumnos destacaron la pertinencia de su uso para mejorar sus competencias de escritura, para tener mayor claridad respecto a lo que se espera de ellos en los trabajos y para que se establezcan condiciones más objetivas de evaluación:

Al tener la rúbrica y conocerla desde el principio se puede ir verificando si se cumple con la guía y no hay sorpresas en la evaluación. (GDE2).

Tenemos un curso [diferente al de Tc] en que entregamos trabajos... [la profesora] los califica, pero nunca nos entrega nada, entonces, bueno saqué tres, pero... ¿qué me quedó malo o por qué saqué esto?... mientras que en este curso tenemos los criterios y ya sabemos por qué nos quedó mal tal o cual punto. (GDE5).

Establecer los criterios de evaluación también fue un elemento importante para los profesores, pues facilitó el proceso de calificación y aportó a una valoración más objetiva y formativa de los textos.

La rúbrica ayuda mucho, no sólo para que los muchachos sepan qué se espera de ellos, sino para que uno mismo deje de evaluar intuitivamente. Obliga a hacer el esfuerzo de pensar qué es lo que realmente se va a tener en cuenta en la calificación. (P1).

La rúbrica de calificación permite que el proceso sea más sencillo. Al principio uno no está familiarizado, pero a medida que la usa le encuentra utilidad y sabe que es pertinente tanto para uno como para ellos. (P2).

\subsection{Incidencia en el aprendizaje}

Esta categoría se refiere a los cambios identificados en las representaciones de los estudiantes sobre la escritura y en sus prácticas de composición a partir de la estrategia de trabajo colaborativo docente. Entre las características de los estudiantes cabe resaltar que se encontraban en semestres avanzados en su proceso de formación (entre quinto y séptimo semestre), con una edad promedio de 20 años y en un rango entre 18 y 33, características que les permitían establecer comparaciones entre las asignaturas con TC y otras asignaturas.

Representaciones de los estudiantes sobre el propósito de la escritura. Esta categoría responde a cuál es el propósito de la escritura en la universidad. En las encuestas realizadas al inicio del semestre (figura 5) los estudiantes identificaron como principal propósito la certificación de saberes (82\%), seguida por la comunicación de ideas (65\%) y, en menor medida, establecieron relaciones entre la escritura y el aprendizaje de contenidos en los cursos (21\%). En la encuesta al final del semestre se observan cambios importantes en la identificación de los propósitos, pues aumentaron los porcentajes en aprendizaje de contenidos (88\%) y comunicación de ideas (93\%). 


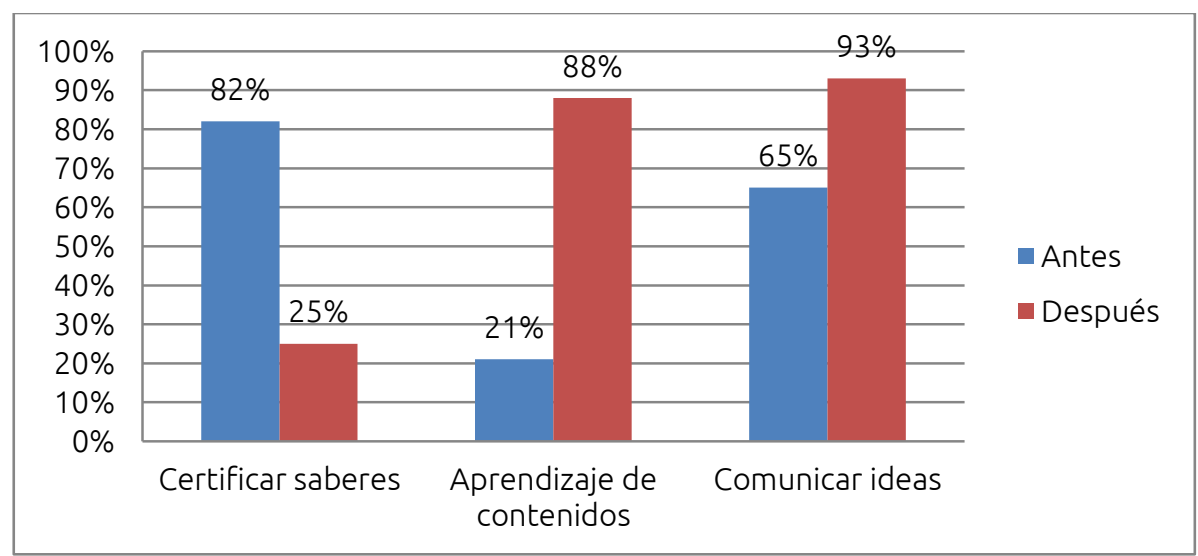

Fuente: elaboración propia.

Figura 5. Propósito de la escritura en la universidad.

En el grupo de discusión se reitera la relación entre el aprendizaje y la escritura:

[La escritura] sirve para aprender... antes de que tú vayas a escribir debes haber entendido algo, si no lo has entendido no vas a poder escribir... en el momento en que lo vas a escribir es que te vas a dar cuenta si de verdad lo tienes así de claro. (GDE1).

(...) uno muchas veces tiene todas las ideas en la cabeza y tiene clarísimo qué es lo que va a escribir... después le deja un tiempo y lo lee y no concuerda lo que uno está escribiendo con lo que uno estaba pensando... entonces se da cuenta que no aprendió. (GDE2).

Prácticas de composición escrita de los estudiantes. Esta categoría buscaba identificar las prácticas de composición escrita de los estudiantes antes y después de la estrategia de trabajo colaborativo. En la encuesta inicial se pidió a los estudiantes que indicaran la frecuencia con que seguían las tres fases fundamentales del proceso de composición: planeación, redacción y revisión.

Respecto a la planeación, es decir, la fase de generación y organización de las ideas, en la encuesta inicial la mitad de los estudiantes la identificó como una práctica muy poco frecuente, mientras que en la encuesta final el 65\% indicó que es una práctica que siempre lleva a cabo al escribir.

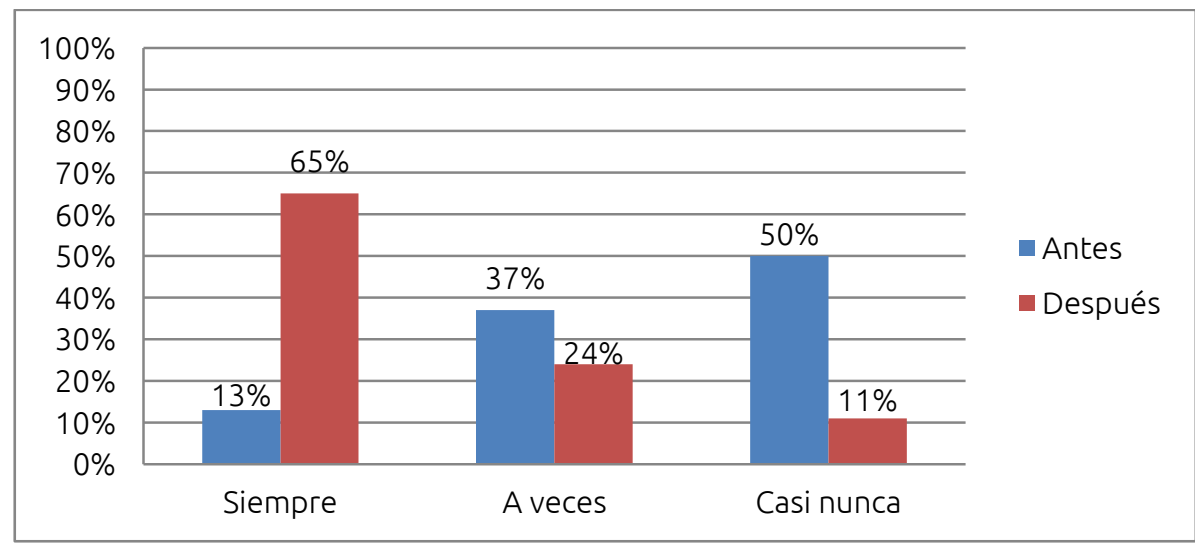

Fuente: elaboración propia.

Figura 6. Práctica de planeación de los textos.

La fase de redacción no presentó variaciones, debido a que en ella se materializa la escritura. La fase de revisión se identificó como más frecuente que la planeación en la encuesta inicial (figura 7) y en la 
encuesta final el 70\% de los estudiantes indicó que siempre la lleva a cabo al escribir.

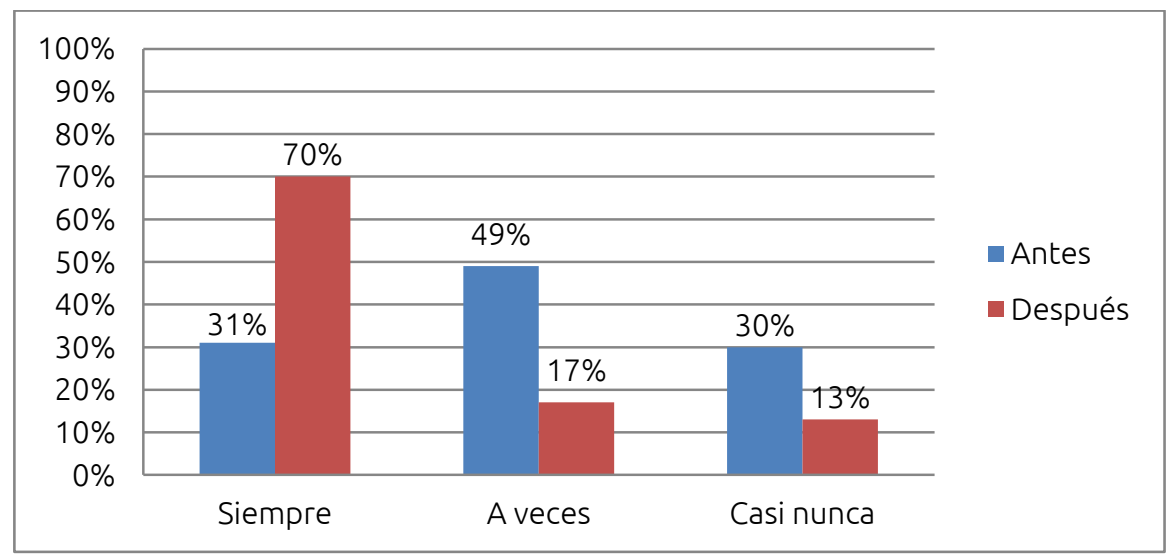

Fuente: elaboración propia.

Figura 7. Práctica de revisión de los textos.

Al respecto, en el grupo de discusión se evidencian las relaciones entre las prácticas de orientación de los profesores y las prácticas de composición de los estudiantes. En las cuatro asignaturas se solicitó la realización de la planeación, se compartieron criterios de evaluación con los estudiantes y se entregaron avances antes de la versión definitiva.

La lógica de todo proceso es la misma, primero se piensa, luego se hace y luego se evalúa, pero con la escritura a veces se nos pasan estos pasos, por eso que el mismo profesor lo pida es una ventaja, uno empieza a organizar la información para un borrador, sigue escribiendo, si tiene los criterios de evaluación trata de aplicarlos. (GDE 4).

Una de las profesoras indicó que los estudiantes van transformando sus prácticas de composición y piden al docente ser coherente con el proceso orientado:

(...) la gente al principio está reacia y ya después está pidiéndote criterios de evaluación... Luego quieren que se entregue al menos un borrador, quieren saber si el esquema inicial va bien encaminado. (P4).

Por último, en esta categoría se indagó con los estudiantes acerca de los aspectos a los que más prestan atención en sus escritos.

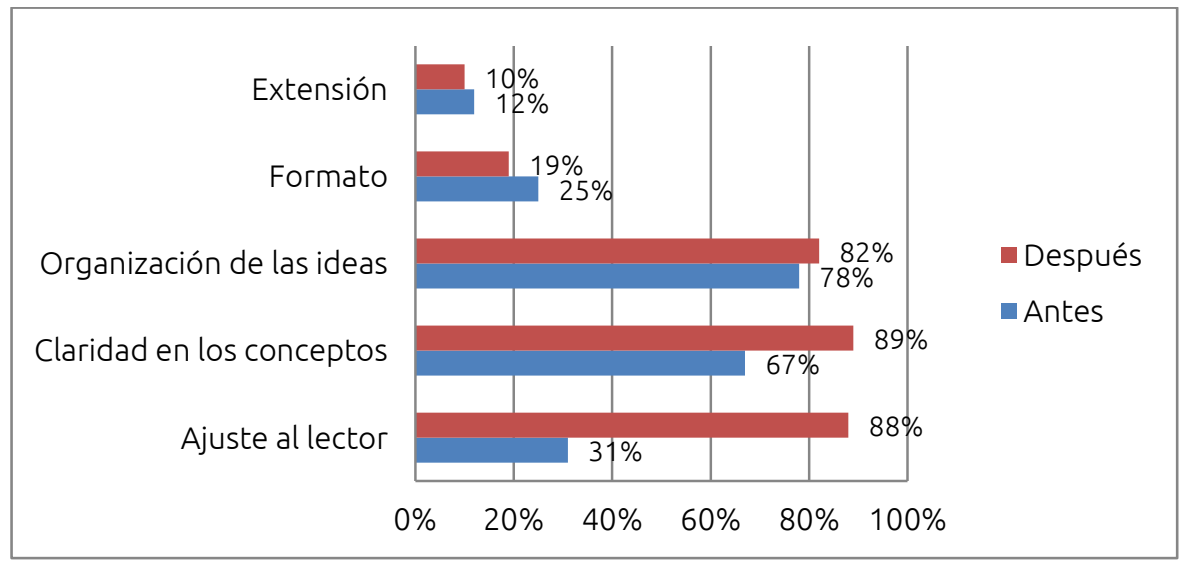

Fuente: elaboración propia.

Figura 8. Aspectos privilegiados en la escritura. 
Como puede evidenciarse en la figura 8, tanto en la encuesta inicial como en la final los estudiantes indicaron que privilegian aspectos de la escritura como la organización de las ideas y la claridad en los conceptos. El principal cambio se dio en el ajuste al lector. Esto se relaciona con las demandas planteadas en las asignaturas a partir del trabajo colaborativo entre el docente de lengua y el docente de la disciplina. En "Procesos de ingeniería de software" escribieron un proyecto, un manual técnico (lector experto) y un manual de usuario (lector lego), en "Títulos valores" dirigieron el texto a un juez, en "Microbiología y cine" a profesionales de la salud y en "Creatividad e idea de negocio" el texto iba dirigido a inversionistas. El ajuste a audiencias particulares constituyó un reto para los estudiantes, como se evidencia en sus intervenciones en el grupo de discusión:

A veces uno no piensa en el otro, eso hace que no escriba bien. Es importante cuando uno sabe a quién le escribe, por ejemplo, en el consultorio jurídico uno le escribe a un consultante de verdad, no al profesor, entonces tiene que hacerse entender. (GDE2).

(...) el problema es cuando hay que hacer entender eso mismo a otra persona [información de un manual técnico], cuando no es un ingeniero, hay que expresarse claramente, hacerlo entendible para el usuario. (GDE5).

\section{Discusión y conclusiones}

Los resultados evidencian una incidencia positiva de la estrategia de trabajo colaborativo docente tanto en la enseñanza como en el aprendizaje de la escritura en los cursos disciplinares estudiados. Respecto a la incidencia en la enseñanza, se destaca la incorporación de la práctica de diseño y uso de guías escritas por parte de los profesores. Las guías funcionaron como mediadoras entre las expectativas de los docentes y los estudiantes, pues la adecuada representación de la tarea es uno de los elementos clave en el proceso de escritura (Castelló, 2002). En esta representación juegan también un papel crucial las asesorías y el acompañamiento del profesor durante el desarrollo de la asignación.

Por otra parte, la presentación de criterios claros de evaluación a través de rúbricas y la posibilidad de entregar versiones previas al texto definitivo aportaron a la evaluación formativa, en la medida en que los estudiantes tuvieron varias oportunidades para construir, autoevaluar y regular la producción de sus textos (Camps y Ribas, 1993; Broad, 2003). No obstante, es preciso fortalecer el protagonismo de otros agentes en el proceso de evaluación, ya que en tres de las asignaturas esta función fue asumida únicamente por el docente y sólo en una se aprovechó la creación de la rúbrica para hacer revisión entre pares (coevaluación).

Respecto a la incidencia en el aprendizaje de los estudiantes, se evidencia un cambio importante en sus representaciones a partir de las propuestas derivadas del trabajo colaborativo docente, pues se empiezan a establecer relaciones entre la escritura y el aprendizaje de los contenidos, procesos estrechamente ligados (Carlino, 2005). Esta función de la escritura, denominada función epistémica, se acrecienta aún más con el intento de los alumnos por ajustarse a las características de las audiencias planteadas por sus profesores. Cuando un estudiante organiza los contenidos para una audiencia específica, puede pasar de "decir el conocimiento" a "transformarlo", en palabras de Bereiter y Scardamalia (1992). Además, al complejizarse la escritura, los estudiantes empiezan a asumirla como un proceso con distintas fases y no sólo como un producto.

Estos resultados evidencian la estrecha relación que se establece entre las formas de orientación y evaluación de los docentes y las prácticas de composición que los estudiantes asumen en los cursos (Carlino, 2013). De allí que sea necesario que los profesores cuenten con la formación que les permita transformar su propio quehacer e impactar en las representaciones y prácticas de los alumnos. El trabajo colaborativo docente se presenta entonces como una estrategia de interformación que posibilita, de forma efectiva, el diseño, la orientación y la evaluación de la escritura en los cursos disciplinares. Esta es una estrategia que a gran escala aportaría al desarrollo de la escritura a través del currículo. Este tipo de trabajo requiere de la búsqueda de un objetivo común por parte de los docentes, así como de una negociación constante, establecimiento de relaciones horizontales y estilos comunicativos nutrientes. 
Aunque en esta investigación se evidencia el impacto del trabajo colaborativo en la enseñanza y el aprendizaje de la escritura, sería pertinente identificar su incidencia en las características de los textos producidos por los estudiantes, así como hacer un seguimiento a las transformaciones logradas tanto en las representaciones como en las prácticas de los participantes y su posible transferencia a otros contextos.

\section{Referencias}

Airey, J. (2011). The disciplinary literacy discussion matrix: A heuristic tool for initiating collaboration in higher education. Across the Disciplines, 8(3).

Bardin, L. (1996). Análisis de contenido. Madrid: Akal.

Bereiter, M. y Scardamalia, C. (1992). Dos modelos explicativos de los procesos de composición escrita. Revista Infancia y aprendizaje, 58, 43-64.

Bocca, A. y Vasconcelo, N. (2008). Algunas reflexiones acerca de las prácticas y representaciones sociales en estudiantes universitarios: la escritura académica. Revista Enunciación, 13(1), 20-27.

Broad, R. (2003). What we really value: Beyond rubrics in teaching and assessing writing. Logan, UT: Utah State University Press.

Camps. A. y Ribas, T. (1993). La evaluación del aprendizaje de la composición escrita en situación escolar. Memoria de investigación. España: Ministerio de Educación Cultura y Deporte.

Carlino, P. (2005). Escribir, leer y aprender en la universidad: una introducción a la alfabetización académica. Buenos Aires: Fondo de Cultura Económica.

Carlino, P. (2006). Concepciones y formas de enseñar escritura académica. Revista Signo \& Seña, 16, 71117.

Carlino, P. (2013). Alfabetización académica diez años después. Revista Mexicana de Investigación Educativa, 18(57), 355-381.

Castelló, M. (2002). De la investigación sobre el proceso de composición a la enseñanza de la escritura. Revista signos, 35(51-52), 149-162.

García, M. y Vaillant, D. (2001). Las tareas del formador. España: Aljibe.

Gottschalk, K. y Hjortshoj, K. (2004). The elements of teaching writing. Boston, MA: Bedford/St. Martin's.

Harran, M (2011). Engineering and language discourse collaboration: practice realities. Across the Disciplines, 8(3).

Ibáñez, J. (1986). Más allá de la sociología. El grupo de discusión: técnica y crítica. Madrid: Siglo XXI.

Imbernón, F. (2007). 10 ideas clave. La formación permanente del profesorado: nuevas ideas para formar en la innovación y el cambio. Barcelona: Graó.

Jacobs, C. (2007). Towards a critical understanding of the teaching of discipline-specific academic literacies: Making the tacit explicit. Journal of Education, 41, 59-81.

Kuriloff, P. (2000). The writing consultant: collaboration and team teaching. En S. McLeod y M. Soven (eds.), Writing across the curriculum: a guide to developing programs (94-108). Newbury Park, cA: Sage. 
Lavié, J. (2009). El trabajo colaborativo del profesorado: Un análisis crítico de la cultura organizativa. España: Comunicación Social.

Leibowitz, B., Bozalek, V., Carolissen, R., Nicholls, L., Rohleder, P., Smolders, T. y Swartz, L. (2011). Learning together: lessons from a collaborative curriculum design project. Across the Disciplines, 8(3).

López-Gil, K., Gómez, N. y Pedraza, E. (2012). Taller de diseño de una pauta de evaluación de trabajos escritos desde la perspectiva de alfabetización académica: estudio de caso. Lenguaje, 40(1), 67-98.

López-Noguero, F. (2002). El análisis de contenido como método de investigación. Revista de Educación 21(4), 167-179.

Marshall, D., Conana, H., Maclons, R., Herbert, M. y Volkwyn, T. (2011). Learning as accessing a disciplinary discourse: Integrating academic literacy into introductory physics through collaborative partnership. Across the Disciplines, 8(3). Recuperado de http://wac.colostate.edu/atd/clil/marshalletal.cfm

McLeod, S., Miraglia, E., Soven, M. y Thaiss, C. (Eds.) (2001). WAC for the New millennium: strategies for continuing writing across the curriculum programs. Urbana, IL: National Council of Teachers of English.

McMillan, J. y Schumacher, S. (2005). Investigación educativa. Madrid: Pearson.

Moyano, E. (2010). Escritura académica a lo largo de la carrera: un programa institucional. Revista Signos, 43(74), 465-488.

Olson, B. (2013). Academic writing across the disciplines. literacy design collaborative. Recuperado de https://ldc.org/sites/default/files/LDC academic writing final.pdf

Paretti, M. (2011). Interdisciplinarity as a lens for theorizing language/content partnerships. Across the Disciplines, 8(3).

Pérez, M. y Rincón, G. (Eds.) (2013). ¿Para qué se lee y se escribe en la universidad colombiana? Un aporte a la consolidación de la cultura académica del país. Bogotá: Pontificia Universidad Javeriana.

Tashakkori, A. y Teddlie, C. (Eds.) (2003). Handbook of mixed methods in social and behavioral research. Thousand Oaks, CA: Sage.

Young, A. (2006). Teaching writing across curriculum. Upper Saddle River, NJ: Pearson Education. 